\title{
Phenomenology of Baryon Resonances
}

\section{Michael Döring*}

Department of Physics and Institute for Nuclear Studies, The George Washington University, Washington, DC 20052, USA; Thomas Jefferson National Accelerator Facility, Newport News, VA 23606, USA

E-mail: doringegwu. edu

\section{Justin Landay}

Department of Physics, The George Washington University, Washington, DC 20052, USA

E-mail: justinlanday@gmail.com

Maxim Mai

Department of Physics, The George Washington University, Washington, DC 20052, USA

E-mail: maximmai@email.gwu.edu

\section{Raquel Molina}

Instituto de Física, Universidade de São Paulo, 05508-090 São Paulo, SP, Brazil

E-mail: ramopedif.usp.br

\section{Deborah Rönchen}

Helmholtz-Institut für Strahlen- und Kernphysik (Theorie) and Bethe Center for Theoretical Physics, Universität Bonn, D-53115 Bonn, Germany

E-mail: roenchen@hiskp.uni-bonn.de

Results for light baryon spectroscopy by different collaborations and the state of the art in the subfield is reviewed. Highlights contain common efforts of different phenomenology groups and the impact of recent high-precision data from ELSA, JLab, MAMI, and other facilities. Questions will be addressed, on one side, of how to proceed to reach conclusive answers in baryon spectroscopy, and, on the other side, how phenomenology can be connected to theory in a meaningful way.

XVII International Conference on Hadron Spectroscopy and Structure - Hadron2017

25-29 September, 2017

University of Salamanca, Salamanca, Spain

\footnotetext{
*Speaker.
} 


\section{Introduction}

A key aspect of baryon phenomenology concerns the determination of the resonance spectrum and its properties. In many theoretical approaches to QCD like quark models the resonance spectrum can be calculated but not the amplitudes that could be directly compared to experiment. The phenomenological extraction of resonances bridges this gap allowing theory approaches compare to Nature. Much progress has been reported on this conference, both on the experimental and the phenomenological side. New high-precision data have emerged in recent years and data analyses have much improved covering more reaction channels and energies.

Yet, the determination of the spectrum of excited baryons still bears several challenges. Resonances can be wide and overlapping in energy which makes their detection difficult. Kinematical effects like threshold cusps, threshold openings in the complex plane, or triangle singularities can mimic resonances. Data themselves usually do not cover the full range of energies and scattering angles requiring model-dependent extrapolations. In addition, data from different experiments can show systematic differences that are difficult to reconcile. Amplitude analysis and resonance extraction faces therefore two challenges: The development of an amplitude that respects a maximum of principles from $S$-matrix theory and, second, an improved statistical treatment. While the first topic is subject of ongoing debate, the second one receives less attention. Moreover, the baryon sector is special in the sense that three-body states like $\pi \pi N$ are known to be relevant which makes the analysis conceptually and practically even more challenging.

In Sec. 2 the data aspect is discussed. In particular, it is shown how new high-precision measurements of polarization observables bring results of existing analysis efforts closer together. In Sec. 3 a brief overview of these efforts is provided. More advanced statistical tools for amplitude analyses are discussed in Sec. 4. Sec. 5 is dedicated to theory and model efforts and, in particular, results from lattice QCD in the baryon sector and what is needed to interpret them.

\section{Impact of photoproduction data}

Increasingly accurate data is produced from photoproduction experiments, allowing for much improved amplitude analyses. Double polarization observables are being released that allow to reduce ambiguities and uncertainties of multipole solutions. In addition, more and more final states like $\eta N$ and $K Y$ are measured that can be simultaneously analyzed using multi-channel partialwave analysis techniques. As a result, resonance properties are determined to greater precision allowing for improved comparison with theory.

Yet, the multipoles extracted by different groups differ from each other because different parameterizations are used, the analyzed data bases are different, and the quality of the fits $\left(\chi^{2}\right.$ or likelihood) is different. On top of this, the different groups treat systematic uncertainties differently. Given the spectacular experimental progress in recent years, it becomes, therefore, desirable to address these problems.

To check whether the data themselves can help to reduce discrepancies in different multipole solutions, the SAID, MAID, Bonn-Gatchina and Jülich-Bonn groups teamed up [1] to compare solutions before and after the inclusion of new high-precision $\pi^{0}$ photoproduction data produced 
at ELSA [2, 3, 4, 5] and presented at this conference by U. Thoma. The quality of the fits by the different groups to the new data is similar as shown in Ref. [1].

Comparing the multipoles, the visual inspection already indicates that solutions came closer to each other. We have also quantified this by calculating the pairwise and overall variances between the SAID, Bonn-Gatchina, and Jülich-Bonn solutions before and after the inclusion of the new data. That variance, at a given energy, is defined as the sum over the squared differences in all multipoles up to an angular momentum of the final state of $L=4$. In Fig. 1 the overall variance is shown as

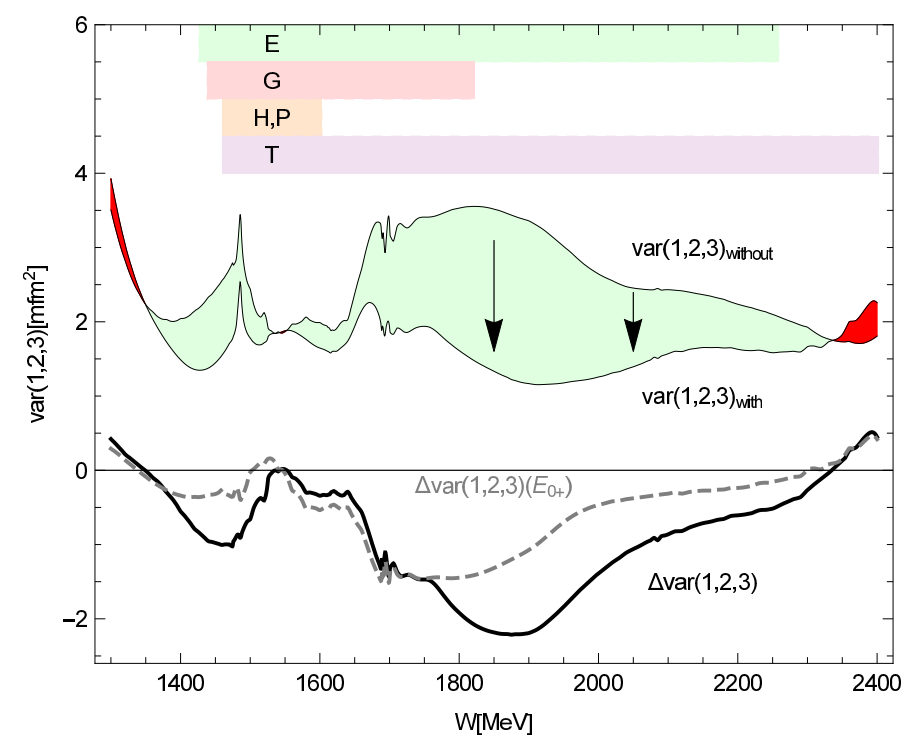

Figure 1: The variance of all three PWAs summed over all $\gamma p \rightarrow \pi^{0} p$ multipoles up to $L=4$ [1]. The range covered by the new double polarization observables $[2,3,4,5]$ are indicated by shaded areas. Over the largest part of the energy range the new data have enforced an improvement of the overall consistency. The improvement is displayed as light green area. The contribution to the improvement from the $E_{0+}$ wave is shown as the dashed curve. Ranges with an overall deterioration are marked in red.

a function of total energy. Indeed, in the energy region covered by the new high precision data, discrepancies are substantially reduced as indicated with the arrows in the upper part of the figure. The solid line in the lower part shows the change, while the dashed line indicates the change from the $E_{0+}$ multipole alone. At lower energies, the solutions come closer to each other even outside the window covered by the new data, while at very low and high energies solutions show slightly larger discrepancies (red areas).

This is a clear indication that indeed high precision data help to determine multipoles more precisely and remove discrepancies between the solutions of different groups. In conclusion, while new high-precision data significantly reduce discrepancies among different partial-wave analysis groups, the details show that it is too early to state perfect matching.

The analysis of photoproduction data could also benefit from improved data on pion-induced reactions, as recently achieved for elastic pion-nucleon scattering [6]. In Ref. [7] the data on the reactions $\pi N \rightarrow \eta N, K Y$ were discussed and analyzed. For example, the measured observables $d \sigma / d \Omega, P$, and $\beta$ for the reaction $\pi N \rightarrow K \Lambda$ provide, in principle, a complete experiment up to a discrete ambiguity. However, the data are in many cases conflicting, in general not very precise, 
or even known to be problematic as in $\pi N \rightarrow \eta N$ at higher energies. Re-measurements of these reactions would be desirable and provide complementary information on the baryon spectrum. Physics opportunities with hadron beams have been discussed in Ref. [8]. A new experiment at J-PARC is dedicated to this effort [9].

\section{Baryon analysis efforts}

To briefly classify different analysis efforts in baryon phenomenology (in a simplified way) consider Fig. 2. For the hadronic interaction one can write the corresponding scattering equation symbolically as

$$
T_{q p}^{i j}=B_{q p}^{i j}+B_{q \ell}^{i k} \tau_{\ell}^{k} T_{\ell p}^{k j}
$$

for incoming, outgoing, and intermediate four-momenta (channels) $p, q, \ell(j, i, k)$, respectively.
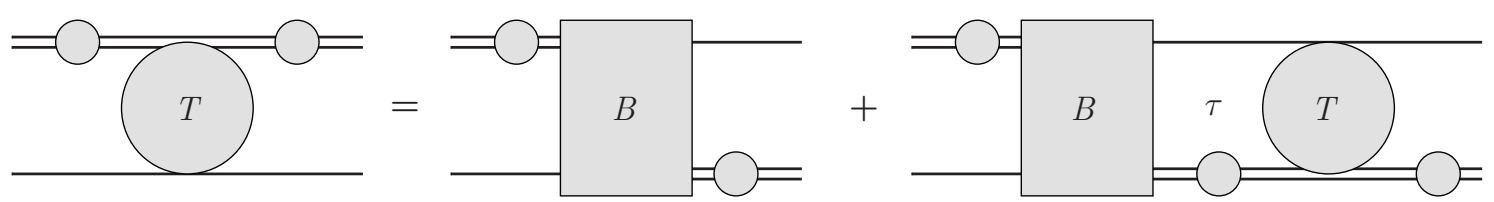

Figure 2: General unitarization scheme of the $T$-matrix. The interaction is denoted as $B$, the intermediate propagation as $\tau$. For three-particle interactions, the double lines indicate isobars and single lines the spectators. In the limit of a narrow resonance isobar, the same graphics serves to illustrate the scattering of two stable particles.

In the second term on the right-hand side the integration over $\ell$ is implicit, as is the summation over all possible intermediate spin configurations. The channel $k$ may contain two or three onshell and off-shell propagating stable particles (the figure shows the three-particle case, but one can understand the two-particle case in the limit of a narrow resonance isobar). This Bethe-Salpeterlike unitarization scheme can accommodate two and three-body coupled-channel unitarity but not crossing symmetry which is an $S$-matrix requirement dropped in most analyses. Three-body unitarity allows one to reduce the four-dimensional equation to a three-dimensional one as recently shown [10], and simultaneously imposes two-body unitarity for the sub-amplitudes represented as isobars by double lines in Fig. 2. Three-body unitarity reduces the four-dimensional scattering equation to a three-dimensional integral equation. In fact, it has been shown in Ref. [10] that the unitary scattering equation can be rewritten such that it manifestly depends only on two-body onshell sub-amplitudes and their extrapolation below threshold, and real-valued three-body forces as presented at this conference by Maxim Mai.

With coupled two-body $(\pi N, \eta N, K \Lambda, K \Sigma)$ and three-body channels $(\sigma N, \pi \Delta, \rho N)$ this framework motivates so-called dynamical coupled-channel approaches. Two major approaches of this kind were discussed, namely the ANL/Osaka analysis [11] and the Julich-Bonn analysis [12, 13]. The ANL-Osaka group has recently extended their efforts to the analysis of electroproduction and neutrino-induced reactions [14] and meson-baryon scattering in the strangeness sector [15]. In Fig. 3 a few selected preliminary results of the Julich-Bonn analysis on $K^{+} \Lambda$ photoproduction are shown. The world data base of this reaction is now included in the analysis. Preliminary results indicate that the $N(1900) 3 / 2^{+}$first claimed by the Bonn-Gatchina (BnGa) group [18, 19] indeed significantly improves the description of kaon photoproduction. 

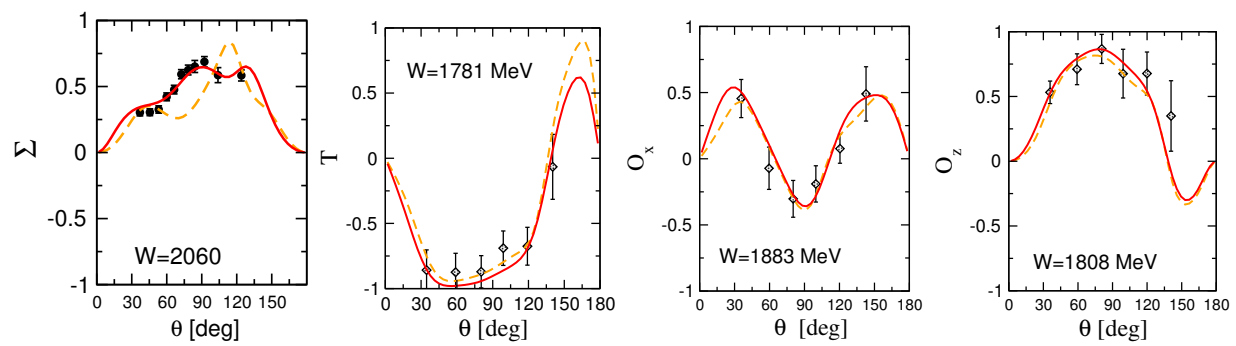

Figure 3: Selected preliminary results of the Julich-Bonn analysis. The dashed lines show the fit without the new CLAS data of Ref. [16], the solid lines the refit including these data. Data are from Refs. [16] ( $\Sigma)$ and [17] $\left(T, O_{x}\right.$ and $\left.O_{z}\right)$.

If the requirement of three-body unitarity on the amplitude is dropped, Eq. (3.1) can be factorized into a matrix equation without sacrifying unitarity for the two-body channels. The interaction $B$ can be either motivated by a Lagrangian framework like in the Giessen approach [20] and in the mentioned dynamical coupled-channel approaches, or phenomenologically with polynomials in energy (contact terms) as in the SAID approach that is characterized by a high degree of model independence because in their Chew-Mandelstam approach resonances are generated from the data itself rather than introduced by hand [21,6]. There, the propagator $\tau$ of Eq. (3.1) contains its real, dispersive part while in $K$-matrix approaches that real part is neglected, representing the simplest possible form of implementing two-body unitarity as realized in the Giessen approach [20]. The BnGa-analysis uses a $K$-matrix formulation but also explores a version including the dispersive parts $[18,19,22]$.

In many analyses a finite number of partial waves is explicitly parametrized and then fitted to data. Sometimes, higher partial waves are taken into account from Regge-inspired $t$ - and $u$ channel exchanges [19]. Cutting the partial-wave space induces some uncertainty, in particular at higher energies. The matching to high-energy dynamics itself poses a problem that can be addressed through finite-energy sum rules as presented by V. Mathieu on this conference. The Joint Physics Analysis Center (JPAC) pursues the high-energy description of baryonic systems and the systematic improvement of matching high- and low-energy physics through such sum rules and related techniques $[23,24,25]$.

Photoproduction reactions have provided new high-precision data as discussed in the previous section. Among the different analysis groups, the $\mathrm{BnGa}$ is the only one systematically analyzing two-meson photoproduction as demonstrated recently, e.g., in Ref. [22]. If the photon becomes virtual, one can study the $Q^{2}$ dependence of the electromagnetic couplings of baryon resonances. This provides a valuable point of comparison for quark models and Dyson-Schwinger calculations. Notably, the data base has much improved through recent Jefferson Lab experiments and their analysis in a unitary isobar framework or the JLS model $[26,27]$. The world data base of electroproduction, with photoproduction reactions as boundary, has been analyzed by the SAID [28] and MAID [29, 30] groups. The upcoming CLAS-12 electroproduction experiments aiming at the discovery of hybrid baryons (LOI12-15-004) predicted by Lattice QCD [31] and at the determination of resonance helicity couplings at high $Q^{2}$ (E12-09-003) to study the transition to the perturbative $\mathrm{QCD}$ regime will require a renewed analysis effort. 
In summary, much progress has been reported for the determination of the baryon spectrum and its properties [32]. The number of new resonances listed by the Particle Data Group [33] has increased and so has the star rating of many states. For a discussion of specific states, see, e.g., Ref. [34]. Many of these new state have been claimed by the BnGa group and evidence for many of them comes from the new high-precision polarization data in photoproduction experiments, e.g., from $K^{+} \Lambda$ photoproduction that acts as isospin filter [16, 35]. Resonances with highly increased significance are, for example, the $N(1875) 3 / 2^{-}, N(1900) 3 / 2^{+}$, and the $N(2060) 5 / 2^{-}$ from the BnGa analysis. The latter two resonances also emerge in the latest fits of the Julich-Bonn group [36].

\section{Statistical aspects}

As discussed, discrepancies among different partial-wave analyses are reduced once highprecision polarization data are included in the analyses. Second, to better judge the uncertainties in a given multi-channel analysis, the inclusion of elastic pion-nucleon scattering can be improved through covariance matrices quantifying the correlations between partial waves [37]. Also, Yannick Wunderlich presented on this conference his work to determine the significant partial waves for many recent photoproduction measurements which can simplify partial-wave analysis significantly [38]. New aspects concerning the complete experiment were discussed in Ref. [39] concerning different truncations for the multipoles. For example, it was shown that all eight $L \leq 2$ multipoles can be reconstructed by measuring only four observables at multiple angles.

Yet, to obtain a reliable spectrum of excited baryons and their properties, it is necessary to make a selection of how many resonance states should be included in the model amplitude. For example, resonances in the SAID approach are generated automatically if required by data and not inserted by hand [21]. The BnGa group uses mass scan techniques by testing new Breit-Wigner type resonances at different energies looking for a minimum of the $\chi^{2}$, preferably in different final states. Yet, one can also demand to select only those models with a minimal resonance content not in contradiction with observation. Model selection can be performed by penalizing the occurrence of resonances through additional terms in the $\chi^{2}$. A very successful method is provided by the socalled least absolute shrinkage and selection operator (LASSO) [40, 41]. However, the size of the penalty is a-priori not determined - obviously, too large of a penalty will remove all resonances from the amplitude while too small of a penalty will lead to an overpopulated spectrum. Therefore, the LASSO penalty parameter needs to be determined by an independent method, in the context of data analysis from cross validation or information theory [41]. Steps in this direction, with the technique applied to low-energy pion photoproduction, have been presented at this conference by Raquel Molina [42] (see also the talk by Boris Grube). With new high precision data from experiment, such automatized methods will become more relevant to increase the evidence of relevant states and discard states with low significance.

\section{Interpretation of the baryon spectrum}

Not each structure in data has to be a resonance that is characterized by a pole in the complex plane of scattering energy. Indeed, cusps effects, complex threshold openings or triangle singulari- 
ties can explain many new phenomena found in recent data. Recent examples are the $\eta^{\prime} p$ threshold cusp observed by the A2 collaboration at MAMI [43], a structure in $K \Sigma$ photoproduction explained as coupled-channel $K^{*} Y$ cusp [44], the $K \Sigma$ cusp in elastic $\pi N$ scattering through new high-precision EPECUR data [6], or a resonance interference and coupled- $K Y$ channel dynamical effect [45, 46] for the structure at around $W \approx 1.68 \mathrm{GeV}$ observed in $\eta$ photoproduction at the proton [47] and the neutron [48]. Several talks at this conference were dedicated to the role of triangle singularities; in the baryon sector, they can be relevant, as well [49]. Another class of non-analyticities that can be misinterpreted as resonance signals are threshold openings in the complex plane of scattering energy as demonstrated in Ref. [50]. In general, a given analysis framework should contain all the mentioned non-analyticities to avoid false positive resonance signals.

Resonances, once established, can be compared to theory predictions. To highlight one of the most emblematic states, the Roper resonance has received much attention. In a recent review [51] progress has been discussed: The unusual $Q^{2}$ dependence of the helicity couplings indicates the intricate dynamics of the Roper that emerges as first radial excitation of the nucleon in quark models. Dyson-Schwinger equations have qualitatively explained the $Q^{2}$ dependence [52], with remaining discrepancies attributed to a "meson cloud". While this can, a posteriori, deliver an improved description of the transition form factor, questions of how to bring models with hadronic degrees of freedom and quark/gluonic degrees of freedom conceptually together remain a challenge. Methods like chiral unitary approaches use only hadrons as degrees of freedom and have been used to study and predict meson-baryon dynamics [53, 54].

Pioneering lattice QCD ab-initio calculations of the baryon spectrum $[55,56]$ have been refined recently $[57,58,59]$, one of the novelties being the determination of eigenenergies with the help of meson-baryon like (5-quark) operators which is important for their reliable extraction [57]. Finite-volume effects are the prime obstacle to interpret these results, especially if pion masses are so low that the $\pi \pi N$ channels can be on-shell. Finite-volume methods for three-body systems are being developed [60, 61] and will be of crucial relevance once lattice QCD calculations in the light baryon sector have reached a similar level as in the light meson sector reported by Raúl Briceño on this conference.

One method is the finite-volume implementation of a three-body unitary amplitude in the finite volume reported by Maxim Mai [60]. As mentioned before, unitarity dictates the imaginary part of the three-particle propagation (indicated as $\tau$ in Fig. 2) but also the isobar-spectator interaction, indicated as $B$ in Fig. 2, if one rewrites the three-body interaction in the isobar formulation [10]. In contrast to two-body scattering, $B$ acquires an imaginary part indicating that the three particles can be simultaneously on-shell while interacting. In the finite volume one distinguishes between the leading, power-law finite-volume effects when particles are on-shell and effects that are exponentially suppressed with volume size and particle mass. To identify the leading effects one can therefore rely on the three-body amplitude of Ref. [10] that determines the imaginary parts and the leading finite-volume effects once adapted to the periodic boundary conditions in the cubic volume.

\section{Summary}

Baryon phenomenology has made much progress in recent years. For energies below $1.7 \mathrm{GeV}$ the baryon spectrum is well established while at higher energies many new states have been found 
mostly from recent high-precision polarization data measured at ELSA, JLab, MAMI and other facilities. The solutions of different baryon analysis groups start to converge and new resonances are being confirmed independently. Yet, discrepancies remain. Currently the implementation of constraints from the high-energy regime promises for a more reliable determination. Non-analyticities other than resonances, like cusps and triangle singularities can explain emergent phenomena. Apart from conceptual improvements of analysis frameworks like three-body unitarity, statistical methods and model selection techniques can help obtain definite answers in the field. Ab-initio lattice QCD calculations have made much progress in recent years, as well. To allow for quantitative comparisons to phenomenology, one problem is tied to the three-body dynamics that is known to be significant in the baryon sector and that induces finite-volume effects subject of current research.

Acknowledgements: M.D. and D.R. gratefully acknowledge the computing time granted on the supercomputer JURECA at Jülich Supercomputing Centre (JSC). This work is also partially supported by the DFG (Deutsche Forschungsgemeinschaft) and NSFC through the Sino-German CRC 110 ("Symmetries and the Emergence of Structure in QCD"). M.M. is supported by a DFG research fellowship (MA 7156/1-1). M.D. is supported by the National Science Foundation (PIF grant No. PHY 1415459 and CAREER grant No. 1452055) and by the U.S. Department of Energy, Office of Science, Office of Nuclear Physics under contract DE-AC05-06OR23177.

\section{References}

[1] A. V. Anisovich et al., The Impact of New Polarization Data from Bonn, Mainz and Jefferson Laboratory on $\gamma p \rightarrow \pi N$ Multipoles, Eur. Phys. J. A 52 (2016) 284 [arXiv:1604.05704 [nucl-th] ].

[2] J. Hartmann et al. [CBELSA/TAPS Collaboration], The polarization observables $T, P$, and $H$ and their impact on $\gamma p \rightarrow p \pi^{0}$ multipoles, Phys. Lett. B $\mathbf{7 4 8}$ (2015) 212 [arXiv: 1506.06226 [nucl-ex]].

[3] A. Thiel et al., Double-polarization observable $G$ in neutral-pion photoproduction off the proton,Eur. Phys. J. A 53 (2017) 8 [arXiv:1604.02922 [nucl-ex] ].

[4] M. Gottschall et al. [CBELSA/TAPS Collaboration], First measurement of the helicity asymmetry for $\gamma p \rightarrow p \pi^{0}$ in the resonance region, Phys. Rev. Lett. 112 (2014), 012003.

[5] A. Thiel et al., Well-established nucleon resonances revisited by double-polarization measurements, Phys. Rev. Lett. 109 (2012) 102001.

[6] A. Gridnev et al. [EPECUR Collaboration], Search for narrow resonances in $\pi p$ elastic scattering from the EPECUR experiment, Phys. Rev. C 93 (2016) 062201 [arXiv:1604.02379 [nucl-ex]].

[7] D. Rönchen et al., Coupled-channel dynamics in the reactions $\pi N \rightarrow \pi N, \eta N, K \Lambda, K \Sigma$, Eur. Phys. J. A 49 (2013) 44 [arXiv:1211.6998 [nucl-th] ].

[8] W. J. Briscoe, M. Döring, H. Haberzettl, D. M. Manley, M. Naruki, I. I. Strakovsky and E. S. Swanson, Physics opportunities with meson beams, Eur. Phys. J. A 51 (2015) 129 [arXiv:1503.07763 [hep-ph]].

[9] K.H. Hicks, H. Sako (spokespersons) et al., 3-Body Hadronic Reactions for New Aspects of Baryon Spectroscopy, approved proposal, http://j-parc.jp/researcher/Hadron/en/pac_1207/pdf/P45_2012-3.pdf 
[10] M. Mai, B. Hu, M. Döring, A. Pilloni and A. Szczepaniak, Three-body Unitarity with Isobars Revisited, Eur. Phys. J. A 53 (2017) 177 [arXiv:1706.06118 [nucl-th] ].

[11] H. Kamano, S. X. Nakamura, T. S. H. Lee and T. Sato, Isospin decomposition of $\gamma N \rightarrow N^{*}$ transitions within a dynamical coupled-channels model, Phys. Rev. C 94 (2016) 015201 [arXiv: 1605.00363 [nucl-th]].

[12] D. Rönchen, M. Döring, H. Haberzettl, J. Haidenbauer, U.-G. Meißner and K. Nakayama, Eta photoproduction in a combined analysis of pion- and photon-induced reactions, Eur. Phys. J. A 51 (2015) 70 [arXiv:1504.01643 [nucl-th]].

[13] D. Rönchen et al., Photocouplings at the Pole from Pion Photoproduction, Eur. Phys. J. A 50 (2014) 101, Erratum: [Eur. Phys. J. A 51 (2015) 63] [arXiv:1401.0634 [nucl-th]].

[14] H. Kamano, S. X. Nakamura, T.-S. H. Lee and T. Sato, Neutrino-induced forward meson-production reactions in nucleon resonance region, Phys. Rev. D 86 (2012) 097503 [arXiv: 1207.5724 [nucl-th]].

[15] H. Kamano, S. X. Nakamura, T.-S. H. Lee and T. Sato, Dynamical coupled-channels model of $K^{-} p$ reactions: Determination of partial-wave amplitudes, Phys. Rev. C 90 (2014) 065204 [arXiv:1407.6839 [nucl-th]].

[16] C. A. Paterson et al. [CLAS Collaboration], Photoproduction of $\Lambda$ and $\Sigma^{0}$ hyperons using linearly polarized photons, Phys. Rev. C 93 (2016) 065201 [arXiv:1603.06492 [nucl-ex]].

[17] A. Lleres et al. [GRAAL Collaboration], Measurement of beam-recoil observables $O_{x}, O_{z}$ and target asymmetry for the reaction $\gamma p \rightarrow K^{+} \Lambda$, Eur. Phys. J. A 39 (2009) 149 [arXiv: 0807.3839 [nucl-ex]].

[18] V. A. Nikonov, A. V. Anisovich, E. Klempt, A. V. Sarantsev and U. Thoma, Further evidence for N(1900) P(13) from photoproduction of hyperons, Phys. Lett. B 662 (2008) 245 [arXiv:0707.3600 [hep-ph]].

[19] A. V. Anisovich, V. Burkert, E. Klempt, V. A. Nikonov, A. V. Sarantsev and U. Thoma, Helicity amplitudes for photoexcitation of nucleon resonances off neutrons, Eur. Phys. J. A 49 (2013) 67 [arXiv:1304.2177 [nucl-ex]].

[20] V. Shklyar, H. Lenske and U. Mosel, $\eta$-meson production in the resonance-energy region, Phys. Rev. C 87 (2013) 015201 [arXiv:1206.5414 [nucl-th]].

[21] R. L. Workman, M. W. Paris, W. J. Briscoe and I. I. Strakovsky, Unified Chew-Mandelstam SAID analysis of pion photoproduction data, Phys. Rev. C 86 (2012) 015202 [arXiv: 1202.0845 [hep-ph]].

[22] V. Sokhoyan et al. [CBELSA/TAPS Collaboration], High-statistics study of the reaction $\gamma p \rightarrow p 2 \pi^{0}$, Eur. Phys. J. A 51 (2015) 95 Erratum: [Eur. Phys. J. A 51 (2015) 187] [arXiv: 1507.02488 [nucl-ex]].

[23] V. Mathieu et al. [JPAC Collaboration], Analyticity Constraints for Hadron Amplitudes: Going High to Heal Low Energy Issues, arXiv:1708.07779 [hep-ph] .

[24] H. Al Ghoul et al. [GlueX Collaboration], Measurement of the beam asymmetry $\Sigma$ for $\pi^{0}$ and $\eta$ photoproduction on the proton at $E_{\gamma}=9$ GeV, Phys. Rev. C 95 (2017) 042201 [arXiv:1701.08123 [nucl-ex]].

[25] J. Nys et al. [JPAC Collaboration], Finite-energy sum rules in eta photoproduction off a nucleon, Phys. Rev. D 95 (2017) no.3, 034014 [arXiv: 1611.04658 [hep-ph] ]. 
[26] I. G. Aznauryan et al. [CLAS Collaboration], Electroexcitation of nucleon resonances from CLAS data on single pion electroproduction, Phys. Rev. C 80 (2009) 055203 [arXiv: 0909.2349 [nucl-ex]].

[27] V. I. Mokeev et al. [CLAS Collaboration], Experimental Study of the $P_{11}(1440)$ and $D_{13}(1520)$ resonances from CLAS data on ep $\rightarrow e^{\prime} \pi^{+} \pi^{-} p^{\prime}$, Phys. Rev. C 86 (2012) 035203 [arXiv:1205.3948 [nucl-ex]].

[28] R. A. Arndt, W. J. Briscoe, M. W. Paris, I. I. Strakovsky and R. L. Workman, Baryon Resonance Analysis from SAID, Chin. Phys. C 33 (2009) 1063 [arXiv:0906.3709 [nucl-th]].

[29] D. Drechsel, S. S. Kamalov and L. Tiator, Unitary Isobar Model - MAID2007, Eur. Phys. J. A 34 (2007) 69 [arXiv:0710.0306 [nucl-th]].

[30] M. Hilt, B. C. Lehnhart, S. Scherer and L. Tiator, Pion photo- and electroproduction in relativistic baryon chiral perturbation theory and the chiral MAID interface, Phys. Rev. C 88 (2013) 055207 [arXiv:1309.3385 [nucl-th]].

[31] J. J. Dudek and R. G. Edwards, Hybrid Baryons in QCD, Phys. Rev. D 85 (2012) 054016 [arXiv:1201.2349 [hep-ph]].

[32] V. Crede and W. Roberts, Progress towards understanding baryon resonances, Rept. Prog. Phys. 76 (2013) 076301 [arXiv:1302.7299 [nucl-ex]].

[33] C. Patrignani et al. [Particle Data Group], Review of Particle Physics, Chin. Phys. C 40 (2016) 100001.

[34] V. D. Burkert et al., Status of some P-wave baryon resonances and importance of inelastic channels, arXiv:1412.0241 [nucl-ex].

[35] T. C. Jude et al. [Crystal Ball at MAMI Collaboration], $K^{+} \Lambda$ and $K^{+} \Sigma^{0}$ photoproduction with fine center-of-mass energy resolution, Phys. Lett. B 735 (2014) 112 [arXiv: 1308.5659 [nucl-ex]].

[36] D. Rönchen et al., publication in preparation.

[37] M. Döring, J. Revier, D. Rönchen and R. L. Workman, Correlations of $\pi N$ partial waves for multireaction analyses, Phys. Rev. C 93 (2016) 065205 [arXiv:1603.07265 [nucl-th]].

[38] Y. Wunderlich, F. Afzal, A. Thiel and R. Beck, Determining the dominant partial wave contributions from angular distributions of single-and double-polarization observables in pseudoscalar meson photoproduction, Eur. Phys. J. A 53 (2017) 86 [arXiv:1611.01031 [physics.data-an]].

[39] R. L. Workman, L. Tiator, Y. Wunderlich, M. Döring and H. Haberzettl, Amplitude reconstruction from complete photoproduction experiments and truncated partial-wave expansions, Phys. Rev. C 95 (2017) no.1, 015206 [arXiv:1611.04434 [nucl-th]].

[40] B. Guegan, J. Hardin, J. Stevens and M. Williams, Model selection for amplitude analysis, JINST 10 (2015) P09002 [arXiv: 1505.05133 [physics.data-an]].

[41] T. Hasti, R. Tibshirani, J. Friedman, The Elements of Statistical Learning: Data Mining, Inference, and Prediction, Springer 2009, second edition.

[42] J. Landay, M. Döring, C. Fernández-Ramírez, B. Hu and R. Molina, Model Selection for Pion Photoproduction, Phys. Rev. C 95 (2017) 015203 [arXiv:1610.07547 [nucl-th]].

[43] V. L. Kashevarov et al. [A2 Collaboration], Study of $\eta$ and $\eta^{\prime}$ Photoproduction at MAMI, Phys. Rev. Lett. 118 (2017) 212001 [arXiv:1701.04809 [nucl-ex]]. 
[44] A. Ramos and E. Oset, The role of vector-baryon channels and resonances in the $\gamma p \rightarrow K^{0} \Sigma^{+}$and $\gamma n \rightarrow K^{0} \Sigma^{0}$ reactions near the $K^{*} \Lambda$ threshold, Phys. Lett. B 727 (2013) 287 [arXiv: 1304.7975 [nucl-th]].

[45] A. V. Anisovich, E. Klempt, B. Krusche, V. A. Nikonov, A. V. Sarantsev, U. Thoma and D. Werthmüller, Interference phenomena in the $J^{P}=1 / 2^{-}$wave in $\eta$ photoproduction, Eur. Phys. $J$. A 51 (2015) 72 [arXiv: 1501.02093 [nucl-ex]].

[46] M. Döring and K. Nakayama, On the cross section ratio $\sigma(n) / \sigma(p)$ in $\eta$ photoproduction, Phys. Lett. B 683 (2010) 145 [arXiv:0909.3538 [nucl-th]].

[47] I. Senderovich et al. [CLAS Collaboration], First measurement of the helicity asymmetry $E$ in $\eta$ photoproduction on the proton, Phys. Lett. B 755 (2016) 64 [arXiv:1507.00325 [nucl-ex]].

[48] L. Witthauer et al. [CBELSA/TAPS Collaboration], Photoproduction of $\eta$ mesons from the neutron: Cross sections and double polarization observable E, Eur. Phys. J. A 53 (2017) 58 [arXiv:1704.00634 [nucl-ex]].

[49] L. Roca and E. Oset, Role of a triangle singularity in the $\pi \Delta$ decay of the $N(1700)\left(3 / 2^{-}\right)$, Phys. Rev. C 95 (2017) 065211 [arXiv: 1702.07220 [hep-ph] ].

[50] S. Ceci, M. Döring, C. Hanhart, S. Krewald, U.-G. Meißner and A. Švarc, Relevance of complex branch points for partial wave analysis, Phys. Rev. C 84 (2011) 015205 [arXiv: 1104.3490 [nucl-th]].

[51] V. D. Burkert and C. D. Roberts, arXiv:1710.02549 [nucl-ex].

[52] D. J. Wilson, I. C. Cloët, L. Chang and C. D. Roberts, Nucleon and Roper electromagnetic elastic and transition form factors, Phys. Rev. C 85 (2012) 025205 [arXiv:1112.2212 [nucl-th]].

[53] M. Mai, P. C. Bruns and U.-G. Meißner, Pion photoproduction off the proton in a gauge-invariant chiral unitary framework, Phys. Rev. D 86 (2012) 094033 [arXiv:1207.4923 [nucl-th]].

[54] P. C. Bruns, M. Mai and U.-G. Meißner, Chiral dynamics of the S11(1535) and S11(1650) resonances revisited, Phys. Lett. B 697 (2011) 254 [arXiv:1012.2233 [nucl-th]].

[55] R. G. Edwards, J. J. Dudek, D. G. Richards and S. J. Wallace, Excited state baryon spectroscopy from lattice QCD, Phys. Rev. D 84 (2011) 074508 [arXiv:1104.5152 [hep-ph]].

[56] G. P. Engel et al. [BGR Collaboration], QCD with Two Light Dynamical Chirally Improved Quarks: Baryons, Phys. Rev. D 87 (2013) 074504 [arXiv:1301.4318 [hep-lat]].

[57] C. B. Lang, L. Leskovec, M. Padmanath and S. Prelovsek, Phys. Rev. D 95 (2017) no.1, 014510 doi:10.1103/PhysRevD.95.014510 [arXiv:1610.01422 [hep-lat]].

[58] A. L. Kiratidis, W. Kamleh, D. B. Leinweber, Z. W. Liu, F. M. Stokes and A. W. Thomas, Search for low-lying lattice QCD eigenstates in the Roper regime, Phys. Rev. D 95 (2017) no.7, 074507 [arXiv:1608.03051 [hep-lat]].

[59] C. W. Andersen, J. Bulava, B. Hörz and C. Morningstar, The elastic I=3/2 p-wave nucleon-pion scattering amplitude and the $\Delta(1232)$ resonance from $N_{\mathrm{f}}=2+1$ lattice $Q C D$, arXiv:1710.01557 [hep-lat].

[60] M. Mai and M. Döring, Three-body Unitarity in the Finite Volume, Eur. Phys. J. A , in print arXiv:1709.08222 [hep-lat].

[61] R. A. Briceño, M. T. Hansen and S. R. Sharpe, Relating the finite-volume spectrum and the two-and-three-particle $S$ matrix for relativistic systems of identical scalar particles, Phys. Rev. D 95 (2017) 074510 [arXiv:1701.07465 [hep-lat]]. 